\title{
Gear predictor of manual transmission vehicles based on artificial neural network
}

\author{
A. M. Wefky, F. Espinosa, M. Mazo, J. A. Jiménez, E. Santiso, \\ A. Gardel \& D. Pérez \\ Department of Electronics, University of Alcala, Spain
}

\begin{abstract}
Nearly all mechanical systems involve rotating machinery (i.e., a motor or a generator), with gearboxes used to transmit power or/and change speed. Concerning vehicles, there is a specific nonlinear relationship between the size of the tires, linear velocity, engine RPM, gear ratio of the differential, and the gear ratio of the transmission. However, for each car there is a specific range of gear ratio of the transmission for each gear. On the other hand, the gear value is an indication of the driver behaviour and the road conditions, therefore it should be considered to establish non-pollutant driving guidelines. In this paper, two novel feed-forward artificial neural network (ANN) models have been developed and tested with the gear as the network output and the velocity of the engine (RPM) and the velocity of the car in $(\mathrm{Km} / \mathrm{h})$ as the network inputs. A lot of experiments were made using two commercial cars. The prediction efficiency of the proposed models is superior (i.e., the generalization mean square error is about 0.005 ). However after testing with two different vehicles, the conclusion is that on one hand the structure of the ANN model is suitable. On the other hand each vehicle has its specific model parameters. This paper shows that it is difficult to develop a universal model that predicts the gear based on the RPM and speed of any car.
\end{abstract}

Keywords: feed-forward artificial neural networks, gear predictor, manual transmission.

\section{Introduction}

The drivetrain system of the automobile engine consists of the following parts: engine, transmission, drive shaft, differential, and driven wheels. Firstly, the 
transmission is a gear system that adjusts the ratio of engine speed or engine regime (RPM) to the vehicle speed. Mainly, it enables the engine to operate within its optimal performance range regardless of the vehicle speed. In a manual transmission, the driver selects the correct gear ratio from a set of possible gear ratios (usually five of six for modern passenger cars). For each gear, there is a specific gear ratio. But an automatic transmission selects this gear ratio by means of an automatic control system.

Secondly, the drive shaft is used on front-engine rear wheel drive vehicles to couple the transmission output shaft to the differential input shaft. However, in front wheel drive automobiles, a pair of drive shafts couples the transmission to the drive wheels through flexible joints known as constant velocity (CV) joints.

Thirdly, the differential has the following three purposes. The first is the right angle transfer of the rotary motion of the drive shaft to the wheels. The second is to allow each driven wheel to turn at a different speed, because the external wheel must turn faster than the internal wheel when the vehicle is turning a corner. The third is the torque increase provided by the gear ratio. The gear ratio also affects fuel economy. In front wheel drive cars, the transmission, differential, and drive shafts are known collectively as the transaxle assembly. The combination of drive shaft and differential completes the transfer of power from the engine to the rear wheels [1].

Finally, the car's tires can almost be thought of as a third type of gearing. In other words, if the circumference of the tires is L, then for every complete revolution of the wheel, the car travels L meters. Eqn. (1) shows the formula relating the overall gear ratio (i.e., gear ratios of the transmission $\left(\mathrm{gr}_{\mathrm{t}}\right)$ and differential $\left.\left(\mathrm{gr}_{\mathrm{d}}\right)\right)$, the size of the tires $\left(\mathrm{C}_{\mathrm{t}}\right)$, the speed of the car $\left(\mathrm{v}_{\mathrm{c}}\right)$, and the engine speed $\left(\mathrm{v}_{\mathrm{e}}\right)$. The overall gear ratio $\mathrm{gr}_{\mathrm{o}}$ is the product of $\mathrm{gr}_{\mathrm{d}}$ and $\mathrm{gr}_{\mathrm{t}}[2]$.

$$
g r_{o}=g r_{d} * g r_{t}=C_{t} * \frac{v_{e}}{v_{c}}
$$

One of the challenges of the MIVECO research project, in which the authors are involved, is to establish a relationship between the driver behaviour and the road conditions with the non-pollutant driving guidelines. Consequently, the knowledge of the gear value and its relationship with the gases measurement is required. However, in most engine control systems it's difficult to find a sensor for the transmission gear selector position.

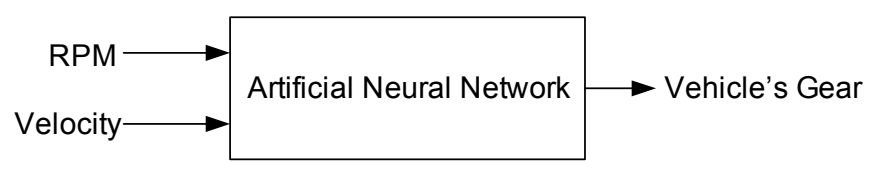

Figure 1: $\quad$ Procedure used to test and train the ANN.

This paper proposes a novel artificial neural network (ANN) model to predict the overall gear ratio $\mathrm{gr}_{\mathrm{o}}$ based on the engine RPM and the corresponding speed 
of the car, see Figure 1. The model has been trained, validated, and tested with experimental tests using two commercial vehicles: Peugeot 205 and 405.

ANNs have been used widely in recent years in various fields such as finance [3], medicine [4], industry [5] and engineering [6, 7], due to their computational speed, their ability to handle complex non-linear functions and their robustness and great efficiency, even in cases where full information for the studied problems is absent.

\section{Methodology}

Figure 2 shows the process followed to evaluate the capability of a feedforward artificial neural network to predict the gear based on the corresponding velocity of the car and RPM of the engine. That procedure was applied to two different vehicles, as explained in the following sections.

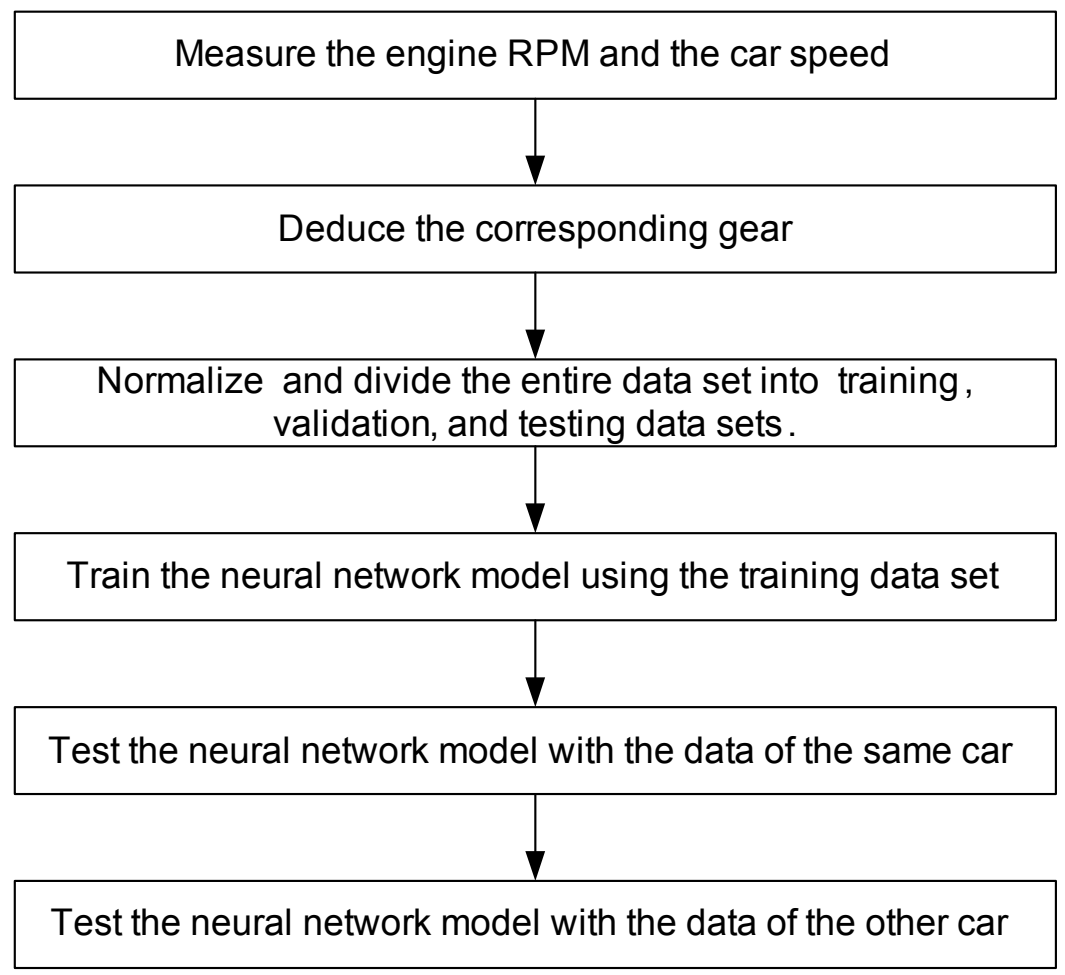

Figure 2: $\quad$ Procedure used to test and train the ANN.

\subsection{First case of study: Peugeot 205}

The engine RPM and the car speed, shown in Figure 3, as well as the overall gear ratio $\mathrm{gr}_{\mathrm{o}}$ were measured in different zones with different driving conditions. The instantaneous values of the gear were calculated where there is a specific 
range of the overall gear ratio for each gear as illustrated in Table 1. The overall gear ratio, the corresponding gear signals, and the filtered gear signal are shown in Figure 4. The horizontal dashed lines in the graph of the overall gear ratio represent the boundaries of the ranges indicated in Table 1 . The overall gear ratio and therefore the gear signal oscillates back and fourth around some boundaries in some areas marked by ellipses. Obviously, these oscillations in the gear signal happen in a very short time period which is impossible in reality. Consequently the gear signal was filtered in order to get rid of these repetitive changes.

Table 1: Ranges of $\mathrm{gr}_{\mathrm{o}}$ with the corresponding gear.

\begin{tabular}{|l|l|l|}
\hline Range of the overall gear ratio $\left(\mathrm{gr}_{\mathrm{o}}\right)$ & \multirow{2}{*}{ Gear } \\
\hline From & To & Neutral \\
\hline 20 & -- & First \\
\hline 11,87628 & 20 & Second \\
\hline 6,74544 & 11,87628 & Third \\
\hline 4,87968 & 6,74544 & Fourth \\
\hline 3,83916 & 4,87968 & Fifth \\
\hline 3,12156 & 3,83916 & \\
\hline
\end{tabular}

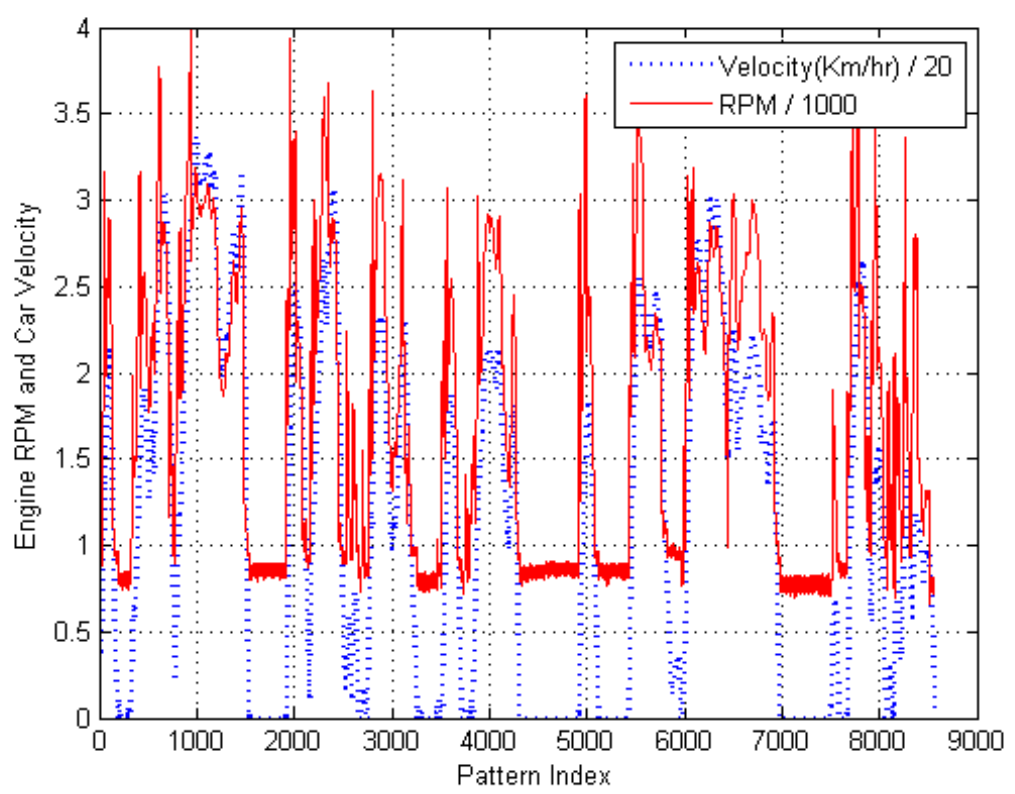

Figure 3: $\quad$ Engine RPM and car velocity of the PEUGEOT 205.

\subsection{Second case of study: Peugeot 406}

In INSIA laboratories in Madrid, the engine RPM was measured during a test of the New European Driving Cycle (NEDC) on rolling roads. The NEDC consists 
of four repeated ECE-15 driving cycles and an Extra-Urban driving cycle (EUDC). The EUDC and only one of the four ECE-15 driving cycles were used. Concerning the speed of the vehicle, the standard values were used. The gear signal was deduced using the clutch signal and the RPM signal. In other words, the gear change event is always synchronized with activating the clutch signal. On the other hand, if the gear changes from a low to a higher position, the RPM level is suddenly decreased. On the contrary, when the gear changes from a high to a lower position, the RPM level decreases smoothly. The instantaneous values of the RPM, reference velocity, clutch, and the deduced gear signals are plotted in Figure 5.
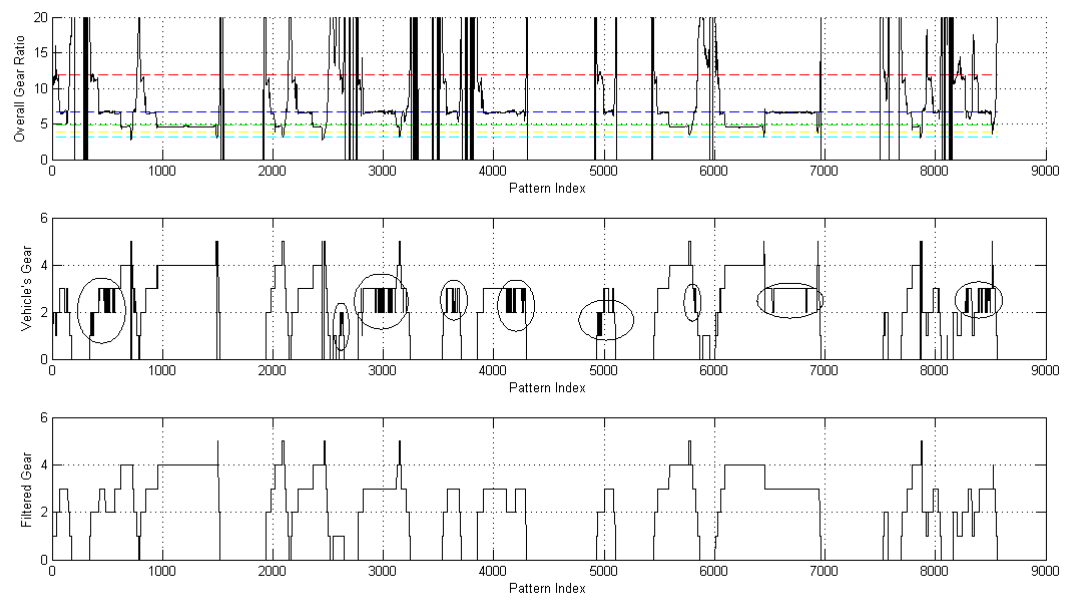

Figure 4: $\quad$ Overall gear ratio, gear, and filtered gear.

\subsection{Artificial neural network modelling}

Numerous neural networks are available for function approximation problems. A multilayer Perceptron MLP feedforward neural network trained with backpropagation was chosen to analyze the data because it has many properties useful for the vehicle gear prediction problem. It can efficiently learn large data sets. To obtain a good generalization, the entire data set was divided into training $(60 \%)$, validation $(20 \%)$, and testing $(20 \%)$ groups. MLPs are more powerful than single layer networks because single layer networks are only able to solve linearly separable classification problems [11]. For example, a single-hidden layer feedforward network with a sufficiently large number of neurons can satisfy the "universal approximation" property [8,12, 13, and 14]. A singlehidden layer neural network (1-S-1), with S sigmoid neurons in the hidden layer and linear neurons in the output layer, can produce a response that is a superposition of S sigmoid functions [11]. Moreover, a single-hidden layer feedforward network with any bounded nonlinear transfer function with $\mathrm{N}-1$ 
hidden neurons can represent any $\mathrm{N}$ input-target relations exactly (with zero error) $[15,16,17$, and 18]. However, a double-hidden layers network can represent any $\mathrm{N}$ input-target relations with a negligible small error using only $(\mathrm{N} / 2)+3$ hidden neurons. This means that a network with 2 hidden layers is better than a network with one hidden layer in terms of number of training samples [15]. Consequently, in this paper both single and double hidden layer networks were used with sigmoid hidden neurons and linear output neurons.

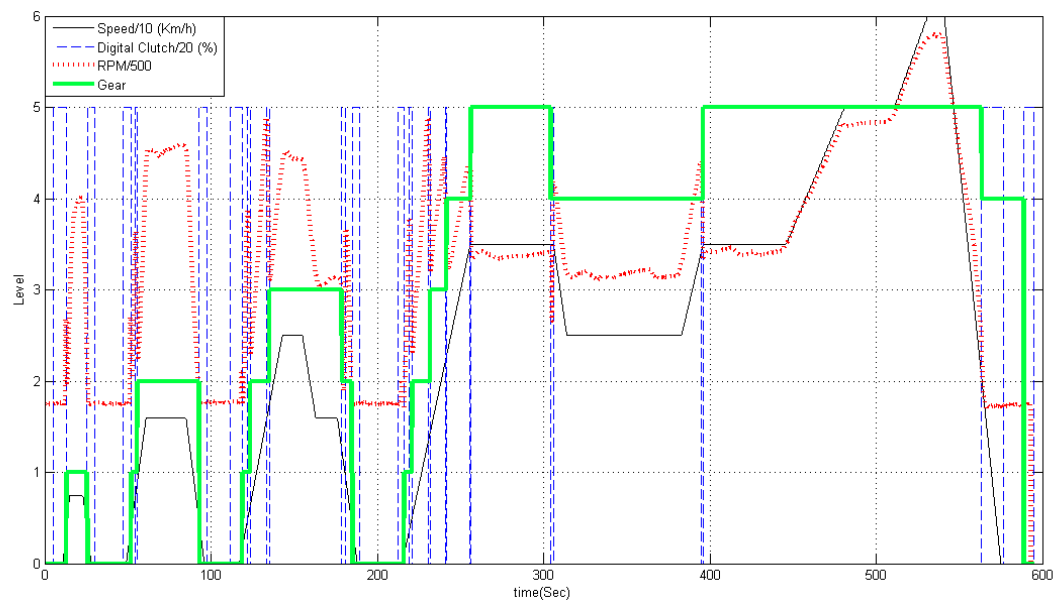

Figure 5: $\quad$ RPM, reference velocity, clutch, and the gear.

When a particular training algorithm fails on a MLP; it could be due to one of two reasons. The learning rule fails to converge to the proper values of the network parameters, perhaps due to unsuitable network initialization. Or the inability of the given network to implement the desired function, perhaps due to insufficient number of hidden neurons. To avoid the first possibility, the neural network models were trained and tested 10 times and the network with the lowest mean square error was chosen. Concerning the second possibility, there is no theory yet to tell you how many hidden neurons are needed to approximate any given function. In most situations, there is no way to determine the best number of hidden neurons without training several networks and estimating the generalization error of each.

However, the designer must put into consideration that the MLP with the minimum size is less likely to learn noise during the training phase; consequently generalizes better to unseen data. The methods to achieve this design objective are: network growing and network pruning. In network growing, we start with a small MLP, and then add a new hidden neuron or new hidden layer when we are unable to meet the design specifications. On the other hand, in network pruning, we start with a large MLP, and then prune it by eliminating certain weights in an orderly manner. If there were too few hidden neurons, high training error and high generalization error would result due to underfitting and high statistical bias. On the other hand, if there were too many hidden neurons, low training 
error, but still have high generalization error, would result due to overfitting and high variance $[7,10,11$, and 13].

\section{Results}

The mean square errors resulting from testing single and double feedforward neural networks with the data of the same vehicle are shown in Tables 2 and 3. The minimum size model architecture that met the design goal (about 0.005) for the PEUGEOT 205 and PEUGEOT 406 was 2-10-1 with 10 hidden neurons.

Table 2: $\quad$ MSE resulting from testing single hidden layer networks.

\begin{tabular}{|l|l|l|}
\hline \multirow{2}{*}{$\begin{array}{l}\text { Number of Hidden } \\
\text { Neurons }\end{array}$} & \multicolumn{3}{|l|}{ Testing Mean Square Error (MSE) } \\
\cline { 2 - 3 } & PEUGEOT 205 & PEUGEOT 406 \\
\hline 1 & 0.022929804113799 & 0.030737847949962 \\
\hline 2 & 0.008113754649602 & 0.013009639928021 \\
\hline & 0.007150878530542 & 0.007953683727784 \\
\hline 5 & 0.006916124765493 & 0.007533362116994 \\
\hline 6 & 0.006770174929462 & 0.007356262805218 \\
\hline 7 & 0.006801600634453 & 0.006821096479489 \\
\hline 8 & 0.007161435831671 & 0.006249000207199 \\
\hline 9 & 0.007262385239038 & 0.005906782786126 \\
\hline 10 & 0.006665429326786 & 0.005840597665814 \\
\hline 11 & 0.006486881856067 & 0.005527620454196 \\
\hline 12 & 0.006699752798641 & 0.004349509945806 \\
\hline 13 & 0.006961214546624 & 0.005195854106452 \\
\hline 14 & 0.006687424328677 & 0.004042310392445 \\
\hline 15 & 0.006733573608595 & 0.005189990010043 \\
\hline
\end{tabular}

Table 3: $\quad$ MSE resulting from testing double hidden layer networks.

\begin{tabular}{|l|l|l|l|}
\hline \multicolumn{2}{|l|}{$\begin{array}{l}\text { Number } \\
\text { neurons }\end{array}$} & of hidden & \multicolumn{2}{l|}{ Testing Mean Square Error (MSE) } \\
\hline S1 & S2 & PEUGEOT 205 & PEUGEOT 406 \\
\hline 1 & 1 & 0.021251738513978 & 0.029045230801033 \\
\hline 2 & 2 & 0.007897066497980 & 0.007546159875325 \\
\hline 3 & 3 & 0.007403216890455 & 0.007741855207261 \\
\hline 4 & 4 & 0.007136833232957 & 0.003671035573348 \\
\hline 5 & 5 & 0.007266126627429 & 0.002963149051319 \\
\hline 6 & 6 & 0.006853332450965 & 0.002682340983058 \\
\hline 7 & 7 & 0.006382907932383 & 0.002440590330384 \\
\hline 8 & 8 & 0.006284173922234 & 0.002303214429815 \\
\hline 9 & 9 & 0.006515939055022 & 0.002130067441743 \\
\hline 10 & 10 & 0.006873106767166 & 0.002144081137843 \\
\hline
\end{tabular}


44 Computational Methods and Experimental Measurements XIV

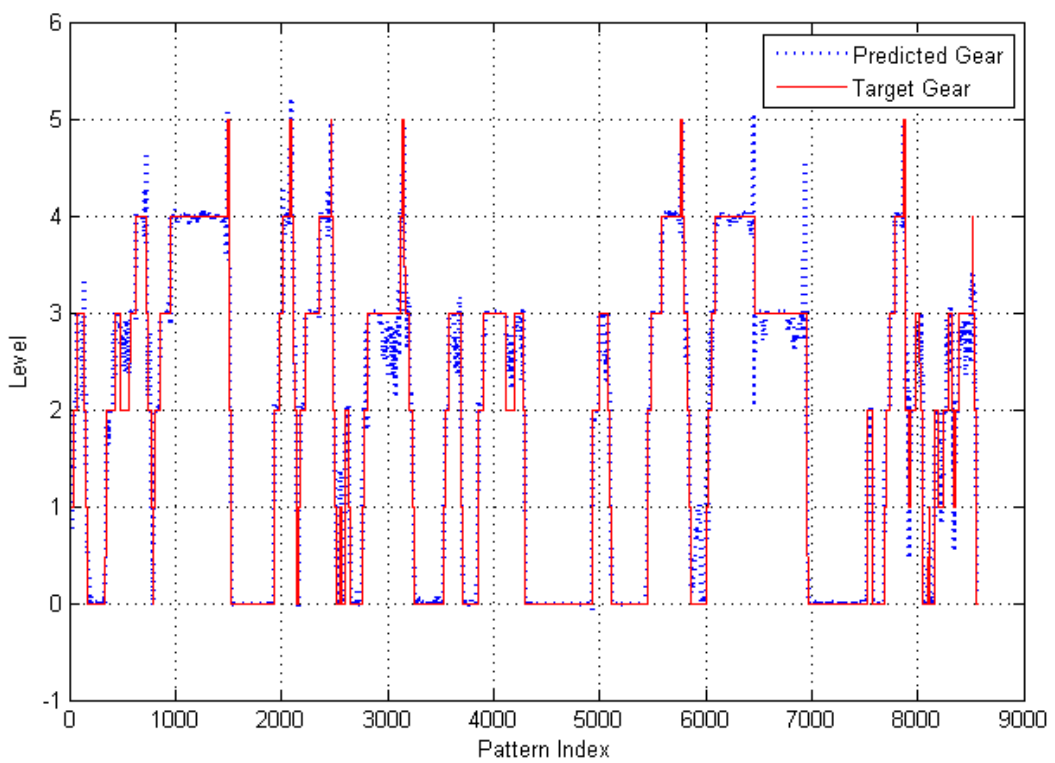

Figure 6: Testing results of the proposed model for P-205 9B.

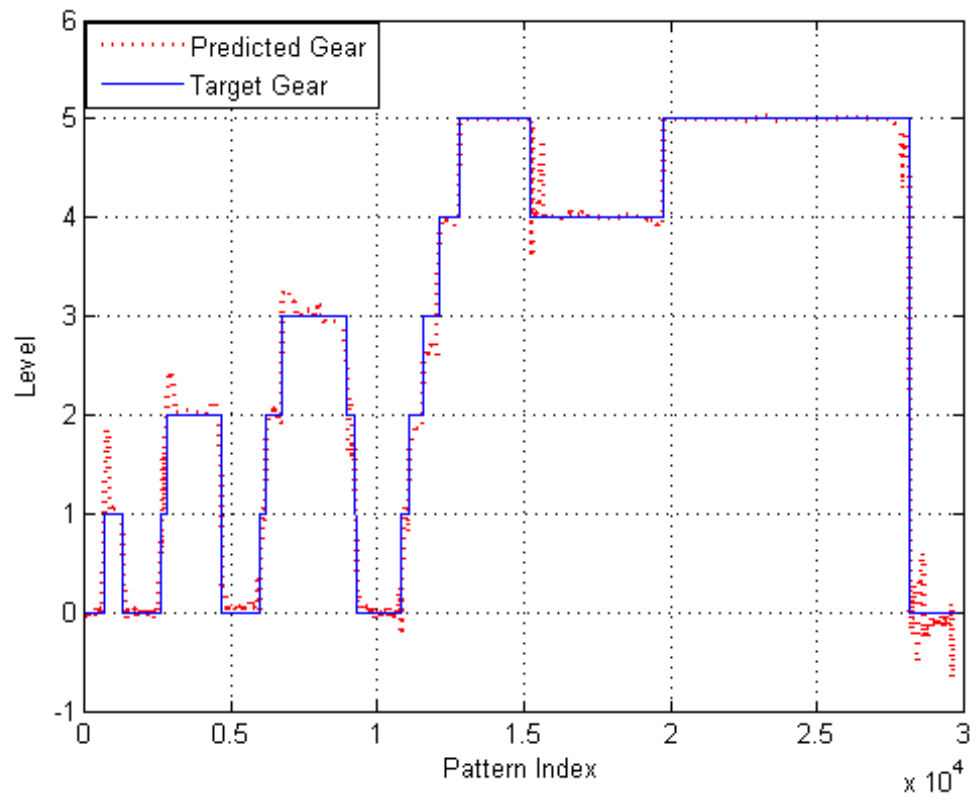

Figure 7: $\quad$ Testing results of the proposed model for P-406. 
The proposed models showed satisfactory results when it was tested with the data of the same vehicle as can be shown in Figures 6 and 7. On the other hand, the models failed to predict the gear signal when they were tested with the data of the other vehicle.

\section{Discussion}

The neural network models succeeded to predict the gear given only the corresponding engine RPM and car speed. Two structures models have been studied. Considering the value of 0.005 for the mean square error as a goal, it can be deduce that the simplest solution is the following structure model: only one hidden layer and 10 neurons per layer. On the other hand the same neural network model failed to predict the gear signal when it was tested with the data of the other vehicle. This means that the parameters (weights and biases) of the model should be calculated for each vehicle.

\section{Conclusion}

An approach to predict the vehicle's gear based on the engine regime (RPM) and the vehicle's velocity $(\mathrm{Km} / \mathrm{h})$ using feedforward neural networks is presented. Two neural network models were evaluated for two different vehicles. The proposed ANN model structure is: only one hidden layer and 11 neurons [10 hidden plus 1 out]. This model allows one an acceptable mean square error (about 0.005 ) to predict the gear of manual transmission vehicles. However, the calculated model parameters for a car cannot be extended to another vehicle; they should be checked for any different case.

\section{References}

[1] Norman P. M., Gerald L., Charles W. Battle, Edward C.J, Understanding Automotive Electronics, 2003, Elsevier Science, USA.

[2] http://en.wikipedia.org/wiki/Main

[3] Y. Bodyanskiy, S. Popov, Neural network approach to forecasting of quasiperiodic financial time series, European Journal of Operational Research, 175(3) (2006) 1357-1366.

[4] M. Frize, C.M. Ennett, M. Stevenson, and H.C.E. Trigg, Clinical decision support systems for intensive care units: using artificial neural networks, Medical Engineering \& Physics 23(3) (2001) 217-225.

[5] M. Sloleimani-Mohseni, B. Thomas, Per Fahlen, Estimation of operative temperature in buildings using artificial neural networks, Journal of Energy and Buildings, 38 (2006) 635-640.

[6] Y.J. Chen, Y.M. Chen, C.B. Wang, H.C. Chu, T.N. Tsai, Developing a multi-layer reference design retrieval technology for knowledge management in engineering design, Expert Systems with Applications, 29(4) (2005) 839-866. 
[7] S. Haykin, Neural Networks: a comprehensive foundation. New York: MacMillan College Publishing Company 1994. O. Nolles, Nonlinear system identification: from classical approaches to neural networks and fuzzy models, Spring-Verlag, Berlin, 2001.

[8] Mukesh Khare, S.M. Shiva Nagendra, Artificial Neural Networks in Vehicular Pollution Modelling, Springer 2007

[9] T. I. Maris, L. Ekonomou, G.P. Fotis, A. Nakulas, E. Zoulias, Electromagnetic field identification using artificial neural networks, Proceedings of the 8th WSEAS International Conference on Neural Networks, Vancouver, British Columbia, Canada, June 19-21, 2007.

[10] Khalaf, A.A.M.; Abo-Eldahab, M.A.M.; Ali, M.M., System Modelling Using Neural Networks in the Presence of Noise, Electronics, Circuits and Systems, 2003. ICECS 2003. Proceedings of the 2003 10th IEEE International Conference on Volume 2, 14-17 Dec. 2003 Page(s):467 - 470 Vol.2.

[11] Martin T. Hagan, Howard B. Demuth, Neural Network Design, 1996 by PWS Publishing Company.

[12] Bishop, C.M. (1995), Neural Networks for Pattern Recognition, Oxford: Oxford University Press.

[13] Sarle, W., 1997. Neural network frequently asked questions. ftp://ftp.sas.com/pub/neural/ FAQ.html.

[14] Guang-Bin Huang; Lei Chen; Chee-Kheong Sien, Universal approximation using incremental constructive feedforward networks with random hidden nodes, Neural Networks, IEEE Transactions on Volume 17, Issue 4, July 2006 Page(s):879 - 892.

[15] S.I. Tamura, M. Tateishi, Capabilities of a four-layered feedforward neural network: four layers versus three", IEEE Transactions on Neural Nets, 8(2) (1997) 251-255.

[16] Sartori, M.A.; Antsaklis, P.J.; A simple method to derive bounds on the size and to train multilayer neural networks, Neural Networks, IEEE Transactions on Volume 2, Issue 4, July 1991 Page(s):467 - 471.

[17] Huang, S.-C.; Huang, Y.-F.; Bounds on number of hidden neurons of multilayer perceptrons in classification and recognition; Circuits and Systems, 1990., IEEE International Symposium on 1-3 May 1990 Page(s):2500 - 2503 vol.4.

[18] Guang-Bin Huang; Babri, H.A.; Upper bounds on the number of hidden neurons in feedforward networks with arbitrary bounded nonlinear activation functions, Neural Networks, IEEE Transactions on Volume 9, Issue 1, Jan. 1998 Page(s):224 - 229. 